\title{
Causal contribution of primate auditory cortex to auditory perceptual decision-making
}

\author{
Joji Tsunada ${ }^{1}$, Andrew S.K. Liu ${ }^{1}$, Joshua I. Gold ${ }^{2,4,}{ }^{*}$, and Yale E. Cohen ${ }^{1,2,3,4}$ \\ 1Department of Otorhinolaryngology, U. Pennsylvania, Philadelphia, PA 19104 \\ ${ }^{2}$ Department of Neuroscience, U. Pennsylvania, Philadelphia, PA 19104 \\ ${ }^{3}$ Department of Bioengineering, U. Pennsylvania, Philadelphia, PA 19104
}

\begin{abstract}
Auditory perceptual decisions are thought to be mediated by the ventral auditory pathway. However, the specific and causal contributions of different brain regions in this pathway, including the middle-lateral (ML) and anterolateral (AL) belt regions of the auditory cortex, to auditory decisions have not been fully identified. To identify these contributions, we recorded from and microstimulated ML and AL sites while monkeys decided whether an auditory stimulus contained more low-frequency or high-frequency tone bursts. Both ML and AL neural activity was modulated by the frequency content of the stimulus. However, only the responses of the most stimulus-sensitive AL neurons were systematically modulated by the monkeys' choices. Consistent with this observation, microstimulation of AL—but not ML—systematically biased the monkeys' behavior toward the choice associated with the preferred frequency of the stimulated site. Together, these findings suggest that AL directly and causally contributes sensory evidence used to form this auditory decision.
\end{abstract}

There is broad consensus that the ventral auditory pathway plays a central role in forming decisions about the identity of auditory stimuli ${ }^{1-5}$. Early stages of this pathway, including the core auditory cortex and two of its prominent targets, the middle-lateral (ML) and anterolateral (AL) belt regions of auditory cortex, encode information relevant to stimulus identity, such as its frequency content. Later stages of this pathway that receive direct and indirect input from ML and AL, including the ventrolateral prefrontal cortex, can encode decision outcomes, such as inferred auditory categories and associated behavioral choices. However, little is known about the process that converts stimulus information represented early in this pathway to the decision outcomes represented later, including which of these

\footnotetext{
Users may view, print, copy, and download text and data-mine the content in such documents, for the purposes of academic research, subject always to the full Conditions of use: http://www.nature.com/authors/editorial_policies/license.html\#terms

Corresponding author: Yale Cohen, Ph.D., Department of Otorhinolaryngology, 3400 Spruce St-5 Ravdin, Philadelphia, PA 19104, Phone: 1215898 7504, ; Email: ycohen@mail.med.upenn.edu

${ }^{4} \mathrm{JIG}$ and YEC contributed equally

AUTHOR CONTRIBUTIONS

JT, ASKL, JIG, and YEC designed the study and wrote the paper; JT and ASKL collected the experimental data; JT, JIG, and YEC analyzed the data.
}

COMPETING FINANCIAL INTERESTS STATEMENT

The authors do not have any competing financial interests. 
early representations constitute the actual evidence used to form a decision ${ }^{3,}{ }^{6} 8$. The goal of this study was to identify whether ML or AL auditory-driven responses are used as evidence to form decisions about the frequency content of auditory stimuli.

To achieve this goal, we recorded and manipulated ML and AL spiking activity in monkeys while they made a difficult decision about whether a noisy stimulus contained more low- or high-frequency tone bursts (Fig. 1). This approach provided three primary benefits ${ }^{9}$. First, we could assess the sensitivity of individual neurons in each brain region to the frequency content of the stimulus and compare such neurometric sensitivity to concurrently measured behavioral sensitivity around psychophysical threshold. These measures can help to identify neural signals that, in principle, could be used to form the decision ${ }^{10}$. Second, we could identify neural signals that were (weakly) modulated by the monkeys' choices for nominally identical stimuli. Under certain conditions, such modulation is expected of neural signals that represent the evidence used to form a perceptual decision ${ }^{9},{ }^{11}$. Third, combined with electrical microstimulation in ML and AL, we could assess the causal contributions of these brain regions to the decision process.

Consistent with previous studies, we found that both ML and AL neurons had frequencytuned responses that, in principle, could be used as sensory evidence to form the auditory decision ${ }^{4},{ }^{8},{ }^{12}{ }^{13}$. By combining these measures with concurrently measured behavioral reports, we advanced previous findings by showing that the AL responses were more closely related to behavior than ML responses. Thus, AL appears to play a more direct, causal role than ML in the formation of this auditory perceptual decision.

\section{RESULTS}

\section{Behavioral performance}

Monkeys T ( $n=52$ sessions) and A ( $n=39$ sessions) reliably reported whether a sequence of tone bursts contained more low-frequency or high-frequency tone bursts on the low-high task, with performance that depended systematically on stimulus coherence (Fig. 2). When a stimulus contained mostly low- or high-frequency tone bursts (coherences near $\pm 100 \%$ ), the monkeys almost always reported the correct answer. This high accuracy for high-coherence stimuli, quantified as low lapse rates (dashed lines in the left panels of Fig. 2), implies that the monkeys were attentive and followed the rules of the task. Their choice accuracy decreased systematically as coherence approached zero; that is, for more difficult stimuli. We quantified this dependence by calculating the monkeys' discrimination thresholds. These discrimination thresholds, which index the steepness of the psychometric (choice) function with respect to coherence and were computed from logistic functions fit to the psychometric data, imply that the monkeys were using relevant information from the auditory stimuli to inform their decisions (blue lines in the left panels of Fig. 2; median [interquartile range, or

IQR] values across sessions were $1.5[1.4-1.8] \frac{\Delta \% \text { high-freq choices }}{\Delta \% \text { coherence }}$ for monkey $\mathrm{T}$ and $1.2[1.0-1.4] \frac{\Delta \% \text { high-freq choices }}{\Delta \% \text { coherence }}$ for monkey A). The monkeys were also relatively unbiased, making roughly equal numbers of low- and high-frequency choices (choice biases, measured as the coherence value corresponding to $50 \%$ high-frequency choices from the 
logistic fits, were $13[-5-31] \%$ coherence for monkey $\mathrm{T}$ and $-22[-30-7] \%$ coherence for monkey A).

A key feature of the low-high task was that on each trial, the monkey, and not the experimenter, controlled the stimulus-presentation duration, thereby providing us with both choice and response-time (RT) data. A primary benefit of this kind of task is that the monkeys' behavior can be used to define the temporal epoch used on each trial to form the decision, thereby facilitating identification of the underlying neural signals ${ }^{14}$. To help better define this epoch, we fit the psychometric and chronometric (RT) data to a drift-diffusion model (DDM) of decision-making (red curves in Fig. 2) ${ }^{15 \_20}$. This model describes the process of forming a decision by temporally accumulating incoming auditory evidence, which we assumed was represented in the brain as the noisy spiking activity of relevant populations of auditory neurons, to one of two pre-defined values. This process is mathematically equivalent to the one-dimensional movement of a particle undergoing Brownian motion to one of two absorbing boundaries. This model accounts for both the choice (which boundary was reached) and the decision time (when the boundary was reached) on each trial. We used the DDM fits to define the decision epoch by subtracting a non-decision time, which was fit as a separate free parameter in the DDM for each of the two choices and included stimulus-encoding and motor-preparation times, from the measured RTs.

Both monkeys' decisions were consistent with a DDM process that interpreted sensory evidence over much of the stimulus-presentation interval, accounting for choices that were both more accurate (Fig. 2, left) and faster (Fig. 2, right) as absolute coherence increased. Like the logistic fits, these DDM fits implied relatively unbiased choices (median [IQR] choice biases across sessions were 14 [-0.7-27] coherence for monkey $\mathrm{T}$ and -7 [-15-4]\% coherence for monkey A; Spearman's correlation coefficient between session-by-session values computed using logistic and DDM fits $=0.96, p=2.7^{*} 10^{-56}$ ) that depended strongly on coherence (median [IQR] discrimination thresholds were

1.6 $[1.4-1.9] \frac{\Delta \% \text { high }- \text { freq choices }}{\Delta \% \text { coherence }}$ for monkey $\mathrm{T}$ and $1.4[1.2-1.6] \frac{\Delta \% \text { high }- \text { freq choices }}{\Delta \% \text { coherence }}$ for monkey A; Spearman's correlation coefficient for DDM versus logistic fits $=0.70$, $p=2.2^{*} 10^{-14}$ ). The DDM fits accounted for the asymmetric RT data in terms of non-decision times, which were larger for high-frequency choices for monkey $\mathrm{T}$ and for low-frequency choices for monkey A and matched our qualitative observations of the monkeys' asymmetric joystick-movement onset times (dashed lines in Fig. 2, right panel).

\section{Recording-site localization}

Because ML and AL are found relatively early in the ventral auditory pathway, we focused on understanding how their spiking activity might provide the auditory evidence used to form an auditory decision later in the pathway (possibly via a DDM-like accumulate-tobound mechanism ${ }^{15}$ ). We classified 140 frequency-tuned single units ( 88 from the right hemisphere of monkey T, 52 from the right hemisphere of monkey A) into ML and AL based on their tonotopic gradients along the anterior-posterior axis (Fig. 3). Similar to previous findings, ML frequency tuning increased at more posterior sites, whereas $\mathrm{AL}$ 
frequency tuning increased at more anterior sites ${ }^{4},{ }^{12},{ }^{13}$. Neurons around the ML-AL border were tuned for low frequencies. Examples of frequency-response profiles with different preferred frequencies are shown in Fig. 3c. The subsequent neurophysiological analyses were conducted on 45 ML neurons (25 from monkey T and 20 from monkey A) and 55 AL neurons (31 from monkey $\mathrm{T}$ and 24 from monkey A). Microstimulation analyses were conducted on $41 \mathrm{ML}$ sites (30 from monkey T and 11 from monkey A) and 44 AL sites (27 from monkey $\mathrm{T}$ and 17 from monkey $\mathrm{A})$. Our findings are robust to uncertainty about the specific location of the ML-AL border (similar results were obtained when we excluded neurons near our estimated border) and when we included multi-unit activity in our analyses (e.g., Supplementary Fig. 1).

\section{Neuronal stimulus sensitivity}

Both ML and AL auditory-driven responses were modulated by the frequency content of the stimulus. For the example ML neuron shown in Fig. 4a, top, the preferred frequency was assigned to the high-frequency value of the tone-burst sequence. Consequently, as signed coherence approached $+100 \%$, the firing rate of the neuron increased. This coherencedependent modulation had a strong phasic increase in activity that started $<\sim 50 \mathrm{~ms}$ after stimulus onset and then persisted for a few hundred ms. For the example AL neuron shown in Fig. 4a, bottom, because the preferred frequency was assigned to the low-frequency value, its firing rate increased as signed coherence approached $-100 \%$. The response of this neuron, like the ML neuron, had coherence-dependent modulations but had slightly later response onsets and more sustained, coherence-dependent responses throughout stimulus presentation. At the population level, both ML and AL showed qualitatively similar trends as these two example units, in both cases showing sensitivity to signed coherence that for ML was most prominent just after stimulus onset but for AL was more persistent throughout stimulus presentation (Fig. 4b).

Despite their slightly different average frequency-dependent response profiles, ML and AL neurons had similar sensitivity to the frequency content of the stimulus (which depended on not just the average response, but also its variability) throughout stimulus presentation. We quantified neuronal sensitivity using ROC-based "neurometric functions" that described the probability that an ideal observer could use the spiking activity of an individual neuron to decide whether a given stimulus contained more high- or low-frequency tone bursts (Supplementary Fig. 2; examples are shown in Fig. 5a) ${ }^{21}$. Across our populations of AL and ML neurons, the slopes of these functions tended to increase from just after stimulus onset until around the time of decision commitment (i.e., the end of the decision time inferred from DDM fits plus an additional $50 \mathrm{~ms}$ to account for the sensory latency; Fig. 5b). The neurometric slopes, which were calculated from firing rates between stimulus onset and the inferred time of the decision commitment, were similar for the two brain regions and the two monkeys (ML,

monkey T: median $[\mathrm{IQR}]=0.6[0.4-0.9] \frac{\Delta \mathrm{ROC} \text { value }}{\Delta \% \text { coherence }}$, monkey A:0.9 $[0.6-1.3] \frac{\Delta \mathrm{ROC} \text { value }}{\Delta \% \text { coherence }}$

; AL, monkey T:0.6 $[0.3-0.8] \frac{\Delta \text { ROC value }}{\Delta \% \text { coherence }} ;$ monkey A:0.8 $[0.4-0.9] \frac{\Delta \text { ROC value }}{\Delta \% \text { coherence }}$

two-tailed Wilcoxon rank-sum test for $H_{0}$ : median difference between ML and AL slopes=0, 
$p=0.46$ for monkey $\mathrm{T}, p=0.13$ for monkey A). Neurometric slopes were slightly lower than the corresponding psychometric slopes for the two brain regions and the two monkeys (median psychometric slope [IQR] from all sessions for both

monkeys $=1.7[1.5-2.0] \frac{\Delta \% \text { high }- \text { freq choices }}{\Delta \% \text { coherence }}$, two-tailed Wilcoxon signed-rank test, monkey T: $p=5.6^{*} 10^{-6} \mathrm{ML}$ and $p=1.2 * 10^{-6} \mathrm{AL}$, monkey A: $p=2.0^{*} 10^{-4} \mathrm{ML}$ and $p=1.8 * 10^{-5} \mathrm{AL}$ ). Thus, on average, single-neuron ML and AL spiking activity was sensitive to stimulus coherence but less so than psychometric sensitivity. This finding implies that either ML or AL activity could, in principle, be pooled to improve sensitivity and provide the evidence needed to make the decision ${ }^{22}$.

There was also a slight session-by-session relationship between neurometric sensitivity and concurrently measured psychometric sensitivity (Fig. 5c-e). We measured this relationship using sliding windows of spike counts measured relative to different task events: (1) the onset of the auditory stimulus, (2) the inferred time of the decision commitment, and (3) the inferred time of movement initiation. Qualitatively, these correlation profiles looked roughly similar in ML and AL: in both cases, they peaked around the inferred time of the decision commitment. However, the effects were statistically more reliable in AL, particularly for data aligned to stimulus onset, suggesting a slightly closer association for AL versus ML activity and perceptual performance (red curves in Fig. 5c). Nevertheless, these results further support the idea that ML and AL stimulus-driven responses were similar, and either or both could, in principle, be used to inform the monkeys' decisions.

\section{Neuronal choice sensitivity}

To more directly assess the relationships between ML and AL activity and the monkeys' decisions, we computed choice probabilities of individual neurons (Fig. 6a). Choice probability quantifies the ability of an ROC-based ideal observer to use spiking activity to discriminate between low- and high-frequency choices for nominally identical stimuli. We found that certain ML and AL neurons were modulated by the monkeys' choices: $20 \%$ (9/45) of ML and 31\% (17/55) of AL neurons had choice-probability values that differed from chance (permutation test for $H_{0}$ : choice-probability value $=0.5, p<0.05$ ). However, because the average value was $\sim 0.5$ in both brain regions (two-tailed Wilcoxon signed-rank test for $H_{0}$ : median=0.5, $p=0.41$ for ML and $p=0.38$ for AL), we did not identify any systematic effect of choice on the spiking activity of our populations of recorded neurons.

Nonetheless, in AL only, we found a strong, positive correlation between choice probability and neurometric sensitivity (i.e., neurometric slope) of individual neurons. That is, the most sensitive AL neurons had task-driven activity that was most related to the monkey's choices $^{23} 26$. This positive correlation, which was found in both monkeys (Fig. 6b-e) and when multi-unit data were also included in the analyses (Supplementary Fig. 1), was evident during stimulus listening (Fig. 6b), around the inferred time of decision commitment (Fig. 6c), and persisted after the inferred time of movement initiation (Fig. 6d). This persistence into the motor-response epoch is consistent with the notion that that choice-related activity during this period reflected feedback from higher decision areas, as opposed to feedforward contributions to the decision itself ${ }^{27}$. We did not find reliable relationships between choice 
probability and neurometric sensitivity for any ML epoch (Fig. 6 and Supplementary Fig. 1). Together, these results imply that AL activity was more related to the monkeys' decisionmaking behavior than ML activity.

\section{Microstimulation}

To identify possible causal roles for each brain region in the low-high task, we applied electrical microstimulation to individual sites in ML ( $n=41$ sites) or AL ( $n=44$ sites) during stimulus presentation and assessed how this manipulation of localized neural activity affected behavior. We found that microstimulation in AL only had a systematic effect on the monkeys' choices: on average, microstimulation biasing the monkeys toward the choice associated with the preferred frequency of the microstimulation site. We could not identify a reliable effect of microstimulation on RT in either brain region.

Four example sites illustrate our main findings (Fig. 7a). ML microstimulation did not have any systematic effect on choice behavior, leaving both the slope (representing sensitivity) and horizontal position (representing a choice bias) of the psychometric function unchanged (Fig. 7a, top). In contrast, AL microstimulation had a systematic effect on choice bias, resulting in more high-frequency choices when applied to a high-frequency site and more low-frequency choices when applied to a low-frequency site, without affecting sensitivity (Fig. 7a, bottom).

These examples suggest that neurons with appropriate frequency tuning in AL, but not ML, provide sensory evidence used to form the decision. According to this interpretation, activation of AL sites tuned for a relatively low frequency should provide more evidence for the low-frequency choice and, therefore, increase the likelihood that the monkey would move the joystick to the right (i.e., ipsilateral to the site of microstimulation, which was in the right hemisphere for both monkeys). Conversely, activation of AL sites tuned for a relatively high frequency should bias movements toward more leftward (contralateral) joystick movements.

Across the population of sites tested, the effects were consistent with this interpretation and not with an alternative possibility that microstimulation simply caused more choices to one side or the other, independent of the frequency tuning of the microstimulation site. Specifically, microstimulation at low-frequency AL sites tended to cause more ipsilateral joystick movements, and microstimulation at high-frequency AL sites tended to cause more contralateral joystick movements (Fig. 7b, bottom). In contrast, there was no systematic effect on choice for either low- or high-frequency microstimulation sites in ML (Fig. 7b, top).

These effects corresponded to systematic shifts of choice biases in the expected direction, based on the frequency tuning of the microstimulation site, for AL but not ML sites (Fig. 7c). On average, ML microstimulation did not cause systematic changes in choice bias (Fig. 7c, top) or discrimination threshold (Fig. 7d, top). In contrast, AL microstimulation shifted choices in the expected direction (Fig. 7c, bottom. The microstimulation effects were too noisy to be sufficiently reliable in each monkey but, as we report, are highly reliable when combining data across monkeys for AL (two-tailed Wilcoxon sign-rank test, $p=0.010$ ) but 
not ML ( $p=0.45)$, and the effects are consistent across the two monkeys for AL (two-factor ANOVA for shift [no microstimulation and microstimulation conditions] $\times$ monkey [monkey $\mathrm{T}$ and monkey A], main effect of shift: $p=0.004$; and the main effect of monkey: $\mathrm{p}=0.53$ ), but not for ML (main effect of shift: $\mathrm{p}=0.29$; and the main effect of monkey: $\mathrm{p}=0.56$ ). Both $\mathrm{ML}$ and AL microstimulation did not affect discrimination threshold (two-tailed Wilcoxon signrank test, $p=0.17$ for ML and $p=0.32$ for AL; Fig. $7 \mathrm{~d}$, bottom).

In principle, one possible reason for the different effects in AL and ML could be systematic differences in the local spatial organization of frequency tuning around sites of microstimulation in the two brain regions, independent of different causal relationships to choice behavior. For example, more homogenous local tuning in AL versus ML might promote more consistent change in sensory evidence provided by AL microstimulation, resulting in more systematic changes in choice behavior. However, two lines of evidence argue against this interpretation. First, for both brain regions, neurons recorded on the same electrode had comparable preferred frequencies (median [IQR] difference in the preferred frequency 0.33 [0-1.2] octaves for ML and 0.33 [0-0.8] octaves for AL; Wilcoxon rank-sum test for $H_{0}$ : ML and AL have the same difference, $p=0.79$ ). Second, we did not identify for either brain region any systematic relationship between the size of the microstimulation current (range $=25-75 \mu \mathrm{Amp}$ ) and the size of the associated shift in the psychometric functions (Pearson's $r$ for current intensity versus psychometric shift $=-0.30, p=0.06$ for $\mathrm{ML}$ and $0.15, p=0.33$ for $\mathrm{AL}$ ) or discrimination threshold ( $r$ for current intensity versus psychometric slope $=-0.06, p=0.72$ for $\mathrm{ML}$ and $0.13, p=0.42$ for $\mathrm{AL}$ ), which might be expected to differ when comparing activation of locally homogenous versus heterogeneous populations. Thus, these microstimulation results suggest that AL plays a more direct and causal role than ML in the formation of the decisions that guided the monkeys' behavioral responses on the low-high task.

\section{DISCUSSION}

We combined behavior, neural recordings, and electrical microstimulation to test if and how auditory-evoked neural responses in the ML and AL belt regions of auditory cortex contribute sensory evidence to a perceptual decision about the frequency content of an auditory stimulus. Despite similar modulation by the timing and frequency content of the stimulus (Fig. 4), ML and AL differed considerably with respect to their relationships to behavioral choices. In particular, neuronal sensitivity was positively correlated with choice probability, and electrical microstimulation biased choices, for AL but not ML. Our results are the first to demonstrate that two distinct brain regions in the ventral auditory pathway can have similar stimulus-driven responses but different causal relationships with a perceptual decision.

Our behavioral analyses suggested that the monkeys formed their auditory decisions by temporally accumulating sensory evidence in a manner consistent with a well-known sequential-sampling model, the DDM ${ }^{18 \_20},{ }^{28}{ }^{33}$. Because ML and AL activity is strongly linked to auditory input and not behavioral output, we focused on analyses that addressed potential roles of these brain regions in providing sensory evidence for the decision. We do not know how and where in the brain this decision is formed, but it is likely that downstream 
brain regions (e.g., the ventrolateral prefrontal cortex ${ }^{34}$ ) might accumulate this evidence to form the decision. On a more practical level, the DDM fits provided an estimate of the time within a trial that the monkeys used to form the decision, allowing us to better identify neural modulations that were relevant to the decision process ${ }^{14}$.

Such modulations included choice-selective responses of the most sensitive AL neurons (i.e., those with the highest neurometric slopes; Fig. 6). One interpretation of this result is that these weak choice-related signals reflect a feedforward decision process that uses pooled activity from the most sensitive AL neurons as evidence ${ }^{22}$, which is consistent with our microstimulation findings and is reminiscent of findings from other systems ${ }^{23} 26$. Alternatively, these signals might represent feedback to relevant sensory regions once the decision is formed elsewhere, which is consistent with our finding that the modulations occurred relatively late in the decision process ${ }^{8},{ }^{27}$. Regardless, this result reinforces the importance of appropriately identifying the temporal window in which to conduct analyses that relate neural activity with behavior ${ }^{14}$. By doing so, we identified striking differences in choice-related signals in ML and AL, which might reflect, in part, their anatomical relationship: because ML provides a major source of auditory information to AL (schematized in Fig. 3), neural representations of stimulus features in ML might be transformed into representations of sensory evidence in AL via a single stage of information processing ${ }^{4}$.

Extending our approaches might also help clarify previous, mixed findings relating auditory cortex to choice behavior. Under certain conditions, neural activity in auditory cortex is not reliably modulated by choice ${ }^{35},{ }^{36}$. Our new findings suggest that such a lack of overall choice modulation might reflect the absence of a direct role in the decision process, like for ML, or alternatively a more nuanced role involving primarily contributions from the mostsensitive neurons, like for AL. In contrast, other conditions have been shown to elicit stronger and more systematic choice-related activity than we found, even for regions in the ventral pathway as early as the primary auditory $\operatorname{cortex}^{8},{ }^{37}$. Several non-exclusive reasons may contribute to this apparent difference from our results. One possibility is basic differences between task designs: we used a forced-choice discrimination task that might require a more complex decision variable, using multiple levels of processing, than the detection tasks used by others ${ }^{8},{ }^{38}$. Second, our task required a relatively high-level decision about the relative frequencies of high and low tone bursts in a sequence, whereas other tasks required decisions about lower-level stimulus features (e.g., pitch or amplitude modulation) that might be represented directly in the responses of individual neurons in the early auditory pathway ${ }^{8}{ }^{39}{ }^{41}$. A third possibility involves differences in how choice-related activity was analyzed. We focused on neural data from trials in which the stimulus was relatively ambiguous (i.e., low coherence) to help isolate the effects of choice from other stimulusrelated factors on neural responses. In contrast, previous studies might have conflated stimulus- and choice-related activity ${ }^{8}, 37,38$.

In summary, our findings are consistent with the hypothesis that the ventral auditory pathway is functionally and causally involved in forming auditory perceptual decisions. A simple, feedforward scheme might involve a representation of the acoustic features of a stimulus in the core auditory cortex and ML, which gets converted into task-relevant sensory 
evidence in AL. This evidence, in turn, is used to form the decision in the ventrolateral prefrontal cortex $^{34}$. The functional contributions of each of these brain regions may be further modified by the specific nature of the task and auditory decision ${ }^{3},{ }^{8}, 37$. This hierarchical relationship may be analogous to that observed for perceptual decisions in the visual and somatosensory systems ${ }^{15}, 42$. Future research should focus on clarifying the differential and specific contributions of core auditory cortex and ML to auditory perception, identifying the mechanism by which sensory evidence in AL is converted into a choice in the prefrontal cortex, and the contribution of correlated activity to perception and decisionmaking ${ }^{8,43}$.

\section{MATERIALS AND METHODS}

The University of Pennsylvania Institutional Animal Care and Use Committee approved all of the experimental protocols. All surgical procedures were conducted under general anesthesia and using aseptic surgical techniques. The authors were not blind to group allocation during the experiment and in assessing the data outcomes.

In each session, a male monkey (Macaca mulatta; monkey T [15 years old] or monkey A [14 years old]) was seated in a primate chair. A calibrated speaker (model MSP7, Yamaha) was placed in front of the monkey at its eye level. The monkey moved a joystick, which was attached to the primate chair, to indicate its behavioral report. These sessions took place in an RF-shielded room that had sound-attenuating walls and echo-absorbing foam on the inner walls.

\section{Identification of Auditory-Cortical Fields}

Prior to recording, the stereotactic locations of AL and ML were identified through structural MRI scans ${ }^{44-46}$. ML and AL were functionally differentiated by their tonotoptic organization ${ }^{4},{ }^{12},{ }^{13}$; see Fig. 3 and the Preferred-frequency task (below).

\section{Auditory Tasks and Stimuli}

Auditory stimuli were generated using the RX6 digital-signal-processing platform (TDT Inc.) and were transduced by the Yamaha speaker.

Preferred-frequency task-Monkeys listened passively while individual tone bursts were presented in a random order. The tone bursts (100-ms duration with a 5-ms $\cos ^{2}$ ramp; $65 \mathrm{~dB}$ SPL) varied between $0.3-12 \mathrm{kHz}$ in one-third octave steps. A neuron's "preferred frequency" was the frequency that evoked the highest firing rate during tone presentation.

Low-high task-This single-interval, two-alternative forced-choice discrimination task required the monkey to report whether a temporal sequence of tone bursts contained more low-frequency or high-frequency tone bursts (Fig. 1). A trial began with the monkey grasping the joystick, and, after a delay of $400 \mathrm{~ms}$, a sequence of tone bursts (50-ms duration; 5-ms $\cos ^{2}$ ramp; 10-ms inter-burst interval) was presented. The monkey moved the joystick: (1) to the right to report that the sequence contained more low-frequency tone bursts, or (2) to the left to report that the sequence contained more high-frequency tone bursts. The monkey could report its choice at any time after stimulus onset. 
The frequency of each tone burst was determined relative to the preferred frequency of a concurrently recorded neuron. By convention, when the preferred frequency was $<1750 \mathrm{~Hz}$, we used it as the "low frequency," and the other (non-preferred) frequency was 1-3 octaves above that value. In contrast, when the preferred frequency was $>1750 \mathrm{~Hz}$, we used it as the "high frequency," and the other (non-preferred) frequency was 1-3 octaves below that value.

On a trial-by-trial basis, we randomly varied the strength of the sensory evidence by varying the proportion of low- and high-frequency tone bursts (coherence) in the auditory stimulus.

A stimulus with all low-frequency tone bursts was a $-100 \%$ coherence stimulus, whereas a stimulus with all high-frequency tone bursts was a $+100 \%$ coherence stimulus. These sequences were the most easily discriminable (Fig. 1a). A stimulus with $0 \%$ coherence was one in which $50 \%$ of the tone bursts were randomly assigned to be low or high frequency. Because this stimulus had equal numbers of low- and high-frequency tone bursts, it was hardest to discriminate (Fig. 1a). Each stimulus was generated by randomly assigning the frequency of each tone burst to the low- or high-frequency value based on the given coherence. For all analyses, stimulus coherence was calculated from the actual proportion of low- and high-frequency tone bursts that were presented from stimulus onset until the monkey indicated its choice by moving the joystick (i.e., during the response-time [RT] interval) on the given trial.

Training procedure and reward schedule for the low-high task-Each monkey was first trained with the most easily discriminable stimuli (i.e., $\pm 100 \%$ coherence stimuli) and given a juice reward for a correct choice. The monkey's reward did not depend on the speed of the behavioral report, only its accuracy. Error trials resulted in a 2-s longer intertrial interval. After performance stabilized, more difficult stimulus coherences were introduced. Because there was not a "correct" answer for a 0\%-coherence stimulus, the monkey received a reward on $50 \%$ of the trials, independent of its behavioral report.

\section{Recording Methodology}

For each recording session, a tungsten microelectrode ( 1.0 M $\Omega$ @ $1 \mathrm{kHz}$; FHC Inc.) was placed in a skull-mounted microdrive (Narishige, MO-95) and then lowered into the brain through a recording chamber. All neural signals were sampled at $24 \mathrm{kHz}$, band-pass filtered between $700 \mathrm{~Hz}$ and $7 \mathrm{kHz}$ (RA16PA and RZ2, TDT Inc.), and stored for online and offline analyses. OpenEx (TDT Inc.), Labview (NI Inc.), and Matlab (The Mathworks Inc.) software synchronized behavioral control with stimulus production and data collection. Single-neuron activity was isolated from the neural signals with on-line (OpenSorter, TDT Inc.) and off-line spike-sorting programs (WaveClus ${ }^{47}$ ).

\section{Data-Collection Strategy}

Once a neuron was isolated, the monkey participated in the preferred-frequency task. After identifying the preferred frequency of the neuron, we assigned the values of the low and high frequencies for the low-high task. Next, the monkey participated in the low-high task.

During electrical-microstimulation sessions, on 50\% of randomly interleaved trials, we delivered negative-leading bipolar current pulses (rate: $300 \mathrm{~Hz}$; pulse duration: $250 \mu \mathrm{s}$; 
amplitude: $25-75 \mu \mathrm{A}$ ) using a dual-output square-pulse stimulator (Grass S88) and two optical isolation units (Grass PSIU6) ${ }^{32}{ }^{48}$. Microstimulation started with stimulus onset and terminated at joystick movement. Microstimulation trials were rewarded using the same schedule as non-microstimulation trials. Thus, the monkeys were not incentivized to respond differently during microstimulation trials than during non-microstimulation trials.

\section{Behavioral Analyses}

Drift-diffusion model—Psychophysical and chronometric data were fit to a standard drift-diffusion model (DDM), which models a decision process in which noisy evidence is accumulated over time until it reaches a fixed bound ${ }^{18} 20,{ }^{29}{ }^{33}$. This version had five free parameters: $\mathrm{k}, \mathrm{A}, \mathrm{B}, \mathrm{F}_{1}$, and $\mathrm{F}_{2}$. $\mathrm{k}$ governed the stimulus sensitivity of the moment-bymoment sensory evidence: the evidence had a Gaussian distribution $N(\mu, 1)$ in which the mean $\mu$ scaled with the stimulus coherence $(\mathrm{COH}): \mu=\mathrm{k} \times \mathrm{COH}$. The decision variable was the temporal accumulation of this momentary sensory evidence. A decision occurred when this decision variable reached a decision bound $(+\mathrm{A}$ or $-\mathrm{B}$, corresponding to a high- and low-frequency choice, respectively). "Decision time" was the time between stimulus onset and the crossing of either bound. "Response time" was the sum of the decision time and a non-decision time ( $F_{1}$ for a high-frequency choice and $F_{2}$ for a low-frequency choice). Nondecision time can include processes such as stimulus encoding and motor preparation. The

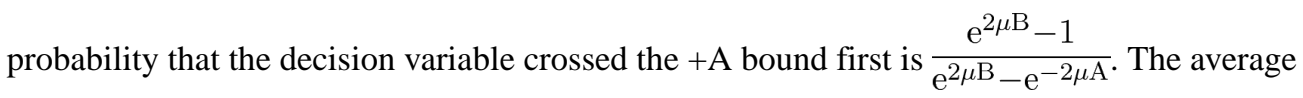
decision time is $\frac{\mathrm{A}+\mathrm{B}}{\mu} \times \operatorname{coth}(\mu(\mathrm{A}+\mathrm{B}))-\frac{\mathrm{B}}{\mu} \operatorname{coth}(\mu \mathrm{B})$ for high-frequency choices and $\frac{\mathrm{A}+\mathrm{B}}{\mu} \times \operatorname{coth}(\mu(\mathrm{A}+\mathrm{B}))-\frac{\mathrm{A}}{\mu} \operatorname{coth}(\mu \mathrm{A})$ for low-frequency choices.

Logistic analysis of psychophysical data-We used a logistic function ${ }^{32,49}, 50$ to fit psychophysical performance during recording and microstimulation sessions ${ }^{31}, 50,51$. This function related the probability (p) that the monkey reported high-frequency choices as a function of coherence $(\mathrm{COH})$ and took the form: $\mathrm{p}=\mathrm{L}+(1-2 \mathrm{~L}) \frac{1}{1+\mathrm{e}^{-\left(\beta_{\mathrm{COH}} * \mathrm{COH}+\beta_{0}\right)}}$. $\mathrm{L}$ represents the upper and lower asymptotes (lapse rates) of the logistic function. $\beta_{\mathrm{COH}}$ represents the effect that coherence had on the monkey's reports; it governs the slope of the psychometric function. $\beta_{0}$ governs the function's horizontal position. A maximum-likelihood procedure fit the logistic function to the behavioral data.

From this logistic fit, we quantified two parameters. (1) Discrimination threshold, a measure of sensitivity to stimulus coherence, depended on $\beta_{\mathrm{COH}}$ and governed the steepness of the psychometric curve (steeper slopes reflected higher sensitivity). Discrimination threshold was defined as the slope of the function determined from the $25 \%$ and $75 \%$ high-frequency choice points ${ }^{10}$. (2) Choice bias depended on $\beta_{0}$ and was the stimulus coherence that elicited $50 \%$ high-frequency choices.

The effect of microstimulation on behavior was quantified using the following procedure. First, we independently fit logistic curves to the behavioral data generated on microstimulation and on non-microstimulation trials, with the assumption of a common 
lapse. Next, using data from non-microstimulation trials, we used a bootstrap procedure to resample trials and fit the resampled data to the logistic function ${ }^{32}$. From this resampling procedure, distributions of discrimination thresholds or choice biases were generated. A microstimulation parameter was considered significant if it was outside the $95 \%$ confidence interval of its resampled distribution.

\section{Neural Analyses}

No statistical methods were used to pre-determine sample sizes but our sample sizes are similar to those reported in previous publications and are similar to those generally employed in the field ${ }^{51}, 52$.

Neurometric analysis-A neurometric function was constructed from neural activity on correct trials for each neuron. We only used correct trials to ensure that the neurometric function indexed stimulus sensitivity, independent of choice effects ${ }^{21},{ }^{22}$. This function plots the probability that an ideal observer could use firing rate alone to correctly identify whether a stimulus contained more high-frequency tone bursts. We computed the curve by comparing neural responses of each neuron to pairs of stimulus coherences with the same magnitude but different signs (e.g., <-80\% versus $>80 \%$ ), which is equivalent to assuming that on a given trial, the decision is made by comparing the responses of a pair of neurons with symmetric tuning properties (i.e., one tuned to the high-frequency value, the other to the low-frequency value) ${ }^{21}$. For each symmetric pair of coherence values, we pooled the firing rates from correct trials into two distributions and generated a receiver-operatingcharacteristic (ROC) curve. The area under the curve is the probability that this ideal observer could discriminate between two stimulus coherences (we expressed this probability as percent correct to facilitate comparison with the psychometric data). This process was repeated for all of the pairs of stimulus coherences $(<-80 \%$ versus $>+80 \% ;-80 \%--40 \%$ versus $+40 \%-+80 \%$; and $-40 \%-0 \%$ versus $0 \%-+40 \%$ ). Finally, this function was fit to a logistic equation analogous to that described in Psychophysical analyses.

Neurometric curves were calculated using 300-ms bins of neural data that advanced in 10$\mathrm{ms}$ increments. Neurometric sensitivity was quantified by calculating the slope of the neurometric function: as the value of $\beta_{\mathrm{COH}}$ of the fitted logistic increases, the slope of the neurometric curve becomes steeper.

Choice probability-Choice probability quantifies the ability of an ROC-based ideal observer to determine choice based only on spike rates from a single neuron, given responses separated by choice (in this case, high frequency versus low frequency) for nominally identical stimulus conditions ${ }^{24}, 26,34,35,53$. We computed choice probability separately for trials using ranges of low-coherence values, which elicited sufficient numbers of correct and error trials to be able to separate responses to a given range into the two choices. The ranges were $-20 \%--10 \% ;-10 \%-0 \% ; 0 \% ; 0 \%-+10 \%$; and $+10 \%-+20 \%$. Reported choice probabilities were the mean of the values computed separately for each range. 


\section{Code availability}

The data analyses were performed in Matlab; this code is available upon request.

A supplementary methods checklist is available.

\section{Supplementary Material}

Refer to Web version on PubMed Central for supplementary material.

\section{Acknowledgments}

We thank Heather Hersh for helpful suggestions on the preparation of this manuscript and Harry Shirley for outstanding veterinary support. This research was supported by grants from the NEI-NIH (JIG), NIDCD-NIH (YEC), and the Boucai Hearing Restoration Fund (YEC).

\section{References}

1. Romanski LM, et al. Dual streams of auditory afferents target multiple domains in the primate prefrontal cortex. Nature neuroscience. 1999; 2:1131-1136. [PubMed: 10570492]

2. Romanski LM, Averbeck BB. The Primate Cortical Auditory System and Neural Representation of Conspecific Vocalizations. Annu Rev Neurosci. 2009; 32:315-346. [PubMed: 19400713]

3. Bizley JK, Cohen YE. The what, where, and how of auditory-object perception. Nat Rev Neurosci. 2013; 14:693-707. [PubMed: 24052177]

4. Hackett TA. Information flow in the auditory cortical network. Hear Res. 2011; 271:133-146. [PubMed: 20116421]

5. Rauschecker JP, Tian B. Mechanisms and streams for processing of "what" and "where" in auditory cortex. Proceedings of the National Academy of Sciences of the United States of America. 2000; 97:11800-11806. [PubMed: 11050212]

6. Giordano BL, McAdams S, Kriegeskorte N, Zatorre RJ, Belin P. Abstract encoding of auditory objects in cortical activity patterns. Cereb Cortex. 2012

7. Rauschecker JP. Ventral and dorsal streams in the evolution of speech and language. Front Evol Neurosci. 2012; 4

8. Niwa M, Johnson JS, O’Connor KN, Sutter ML. Differences between Primary Auditory Cortex and Auditory Belt Related to Encoding and Choice for AM Sounds. J Neurosci. 2013; 33:8378-8395. [PubMed: 23658177]

9. Parker AJ, Newsome WT. Sense and the single neuron: probing the physiology of perception. Annual Review of Neuroscience. 1998; 21:227-277.

10. Klein SA. Measuring, estimating, and understanding the psychometric function: A commentary. Percept Psychophys. 2001; 63:1421-1455. [PubMed: 11800466]

11. Stuttgen MC, Schwarz C, Jakel F. Mapping spikes to sensations. Front Neurosci. 2011; 5:125. [PubMed: 22084627]

12. Kusmierek P, Ortiz M, Rauschecker JP. Sound-identity processing in early areas of the auditory ventral stream in the macaque. Journal of Neurophysiology. 2012; 107:1123-1141. [PubMed: 22131372]

13. Kusmierek P, Rauschecker JP. Functional specialization of medial auditory belt cortex in the alert rhesus monkey. J Neurophysiol. 2009; 102:1606-1622. [PubMed: 19571201]

14. Cohen MR, Newsome WT. Estimates of the Contribution of Single Neurons to Perception Depend on Timescale and Noise Correlation. J Neurosci. 2009; 29:6635-6648. [PubMed: 19458234]

15. Gold JI, Shadlen MN. The neural basis of decision making. Annu Rev Neurosci. 2007; 30:535574. [PubMed: 17600525]

16. Ratcliff R, Gomez P, McKoon G. A diffusion model account of the lexical decision task. Psychol Rev. 2004; 111:159-182. [PubMed: 14756592] 
17. Smith PL, Ratcliff R. Psychology and neurobiology of simple decisions. Trends Neurosci. 2004; 27:161-168. [PubMed: 15036882]

18. Brunton BW, Botvinick MM, Brody CD. Rats and humans can optimally accumulate evidence for decision-making. Science. 2013; 340:95-98. [PubMed: 23559254]

19. Green CS, Pouget A, Bavelier D. Improved probabilistic inference as a general learning mechanism with action video games. Curr Biol. 2010; 20:1573-1579. [PubMed: 20833324]

20. Mulder MJ, et al. The speed and accuracy of perceptual decisions in a random-tone pitch task. Atten Percept Psychophys. 2013; 75:1048-1058. [PubMed: 23572205]

21. Britten KH, Shadlen MN, Newsome WT, Movshon JA. The analysis of visual motion: a comparison of neuronal and psychophysical performance. Journal of Neuroscience. 1992; 12:4745-4765. [PubMed: 1464765]

22. Shadlen MN, Britten KH, Newsome WT, Movshon JA. A computational analysis of the relationship between neuronal and behavioral responses to visual motion. Journal of Neuroscience. 1996; 16:1486-1510. [PubMed: 8778300]

23. Law CT, Gold JI. Neural correlates of perceptual learning in a sensory-motor, but not a sensory, cortical area. Nature neuroscience. 2008; 11:505-513. [PubMed: 18327253]

24. Britten KH, Newsome WT, Shadlen MN, Celebrini S, Movshon JA. A relationship between behavioral choice and the visual responses of neurons in macaque MT. Visual Neuroscience. 1996; 13:87-100. [PubMed: 8730992]

25. Celebrini S, Newsome WT. Neuronal and psychophysical sensitivity to motion signals in extrastriate area MST of the macaque monkey. Journal of Neuroscience. 1994; 14:4109-4124. [PubMed: 8027765]

26. Gu Y, DeAngelis GC, Angelaki DE. A functional link between area MSTd and heading perception based on vestibular signals. Nature neuroscience. 2007; 10:1038-1047. [PubMed: 17618278]

27. Nienborg H, Cumming BG. Decision-related activity in sensory neurons reflects more than a neuron's causal effect. Nature. 2009; 459:89-92. [PubMed: 19270683]

28. Raposo D, Sheppard JP, Schrater PR, Churchland AK. Multisensory decision-making in rats and humans. J Neurosci. 2012; 32:3726-3735. [PubMed: 22423093]

29. Gold JI, Shadlen MN. Banburismus and the brain: decoding the relationship beteween sensory stimuli, decisions, and reward. Neuron. 2002; 36:299-308. [PubMed: 12383783]

30. Eckhoff P, Holmes P, Law C, Connolly PM, Gold JI. On diffusion processes with variable drift rates as models for decision making during learning. New J Phys. 2008; 10:nihpa49499.

31. Ratcliff R, Van Zandt T, McKoon G. Connectionist and diffusion models of reaction time. Psychol Rev. 1999; 106:261-300. [PubMed: 10378014]

32. Ding L, Gold JI. Separate, Causal Roles of the Caudate in Saccadic Choice and Execution in a Perceptual Decision Task. Neuron. 2012:865-874. [PubMed: 22958826]

33. Shadlen, MN.; Hanks, TD.; Churchland, A.; Kiani, R.; Yang, T. The Speed and Accuracy of a Simple Perceptual Decision: A Mathematical Primer. In: Doya, K.; Ishii, S.; Rao, R.; Pouget, A., editors. Bayesian Brain: Probabilistic Approaches to Neural Coding. MIT Press; Cambridge, MA: 2006.

34. Russ BE, Orr LE, Cohen YE. Prefrontal neurons predict choices during an auditory same-different task. Curr Biol. 2008; 18:1483-1488. [PubMed: 18818080]

35. Tsunada J, Lee JH, Cohen YE. Representation of speech categories in the primate auditory cortex. J Neurophysiol. 2011; 105:2634-2646. [PubMed: 21346209]

36. Lemus L, Hernandez A, Romo R. Neural codes for perceptual discrimination of acoustic flutter in the primate auditory cortex. Proceedings of the National Academy of Sciences of the United States of America. 2009; 106:9471-9476. [PubMed: 19458263]

37. Bizley JK, Walker KM, Nodal FR, King AJ, Schnupp JW. Auditory cortex represents both pitch judgments and the corresponding acoustic cues. Curr Biol. 2013; 23:620-625. [PubMed: 23523247]

38. Niwa M, Johnson JS, O'Connor KN, Sutter ML. Active engagement improves primary auditory cortical neurons' ability to discriminate temporal modulation. J Neurosci. 2012; 32:9323-9334. [PubMed: 22764239] 
39. Znamenskiy P, Zador AM. Corticostriatal neurons in auditory cortex drive decisions during auditory discrimination. Nature. 2013; 497:482-485. [PubMed: 23636333]

40. Bizley, JK.; Walker, DK.; Nodal, FR.; King, AJ.; Schnupp, J. Neural correlates of pitch discrimination during passive and active listening. Society for Neuroscience; Washington, DC: 2011.

41. Nienborg H, Cumming BG. Decision-related activity in sensory neurons may depend on the columnar architecture of cerebral cortex. The Journal of neuroscience: the official journal of the Society for Neuroscience. 2014; 34:3579-3585. [PubMed: 24599457]

42. Hernández A, et al. Decoding a perceptual decision process across cortex. Neuron. 2010; 29:300314. [PubMed: 20435005]

43. Cohen MR, Maunsell JH. Attention improves performance primarily by reducing interneuronal correlations. Nature neuroscience. 2009; 12:1594-1600. [PubMed: 19915566]

44. Romanski LM, Goldman-Rakic PS. An auditory domain in primate prefrontal cortex. Nature neuroscience. 2002; 5:15-16. [PubMed: 11753413]

45. Frey S, Comeau R, Hynes B, Mackey S, Petrides M. Frameless stereotaxy in the nonhuman primate. Neuroimage. 2004; 23:1226-1234. [PubMed: 15528122]

46. Cohen YE, Cohen IS, Gifford GW III. Modulation of LIP activity by predictive auditory and visual cues. Cereb Cortex. 2004; 14:1287-1301. [PubMed: 15166102]

47. Quiroga RQ, Nadasy Z, Ben-Shaul M. Unsupervised Spike Detection and Sorting with Wavelets and Superparamagnetic Clustering. Neural Comput. 2004; 16:1661-1687. [PubMed: 15228749]

48. Hanks TD, Ditterich J, Shadlen MN. Microstimulation of macaque area LIP affects decisionmaking in a motion discrimination task. Nature neuroscience. 2006; 9:682-289. [PubMed: 16604069]

49. Salzman CD, Britten KH, Newsome WT. Cortical microstimulation influences perceptual judgements of motion direction. Nature. 1990; 346:174-177. [PubMed: 2366872]

50. Cox, DR. Analysis of binary data. Methuen; London: 1970.

51. Roitman JD, Shadlen MN. Response of neurons in the lateral intraparietal area during a combined visual discrimination reaction time task. J Neurosci. 2002; 22:9475-9489. [PubMed: 12417672]

52. Selezneva E, Scheich H, Brosch M. Dual Time Scales for Categorical Decision Making in Auditory Cortex. Curr Biol. 2006; 16:2428-2433. [PubMed: 17174917]

53. Purushothaman G, Bradley DC. Neural population code for fine perceptual decisions in area MT. Nature neuroscience. 2005; 8:99-106. [PubMed: 15608633] 
a

$+100 \%$ coherence stimulus
(100\% high-frequency tones)

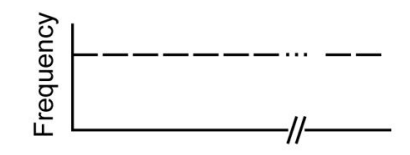

$0 \%$ coherence stimulus ( $50 \%$ high-frequency tones)

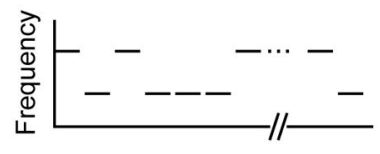

$-100 \%$ coherence stimulus ( $0 \%$ high-frequency tones)

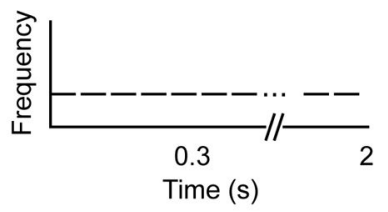

b

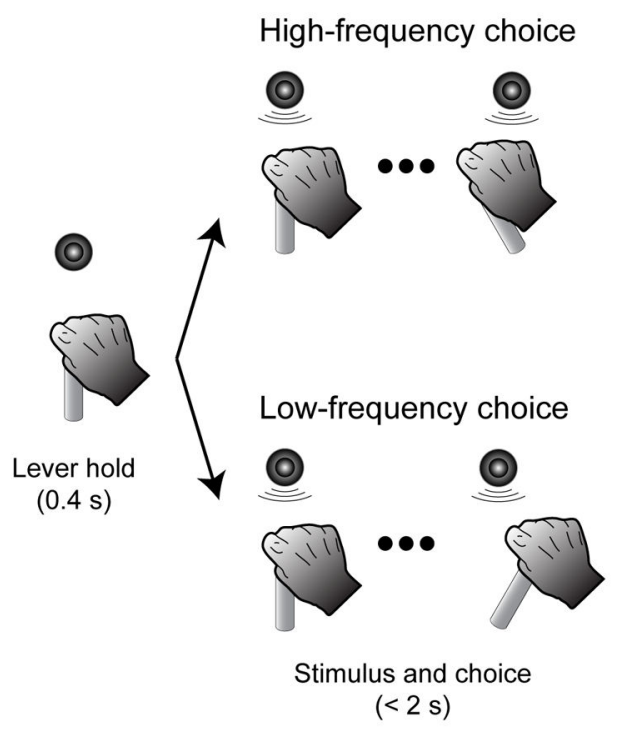

Figure 1. Stimuli and task

a, The auditory stimulus was a temporal sequences of tone bursts. Coherence refers to the percentage of high-frequency bursts (up to $+100 \%$ ) or low-frequency bursts (down to $-100 \%)$. b, The monkey indicated its choice by moving a joystick to the right to report "low frequency" or to the left to report "high frequency." The monkey could report its choice any time after stimulus onset. 

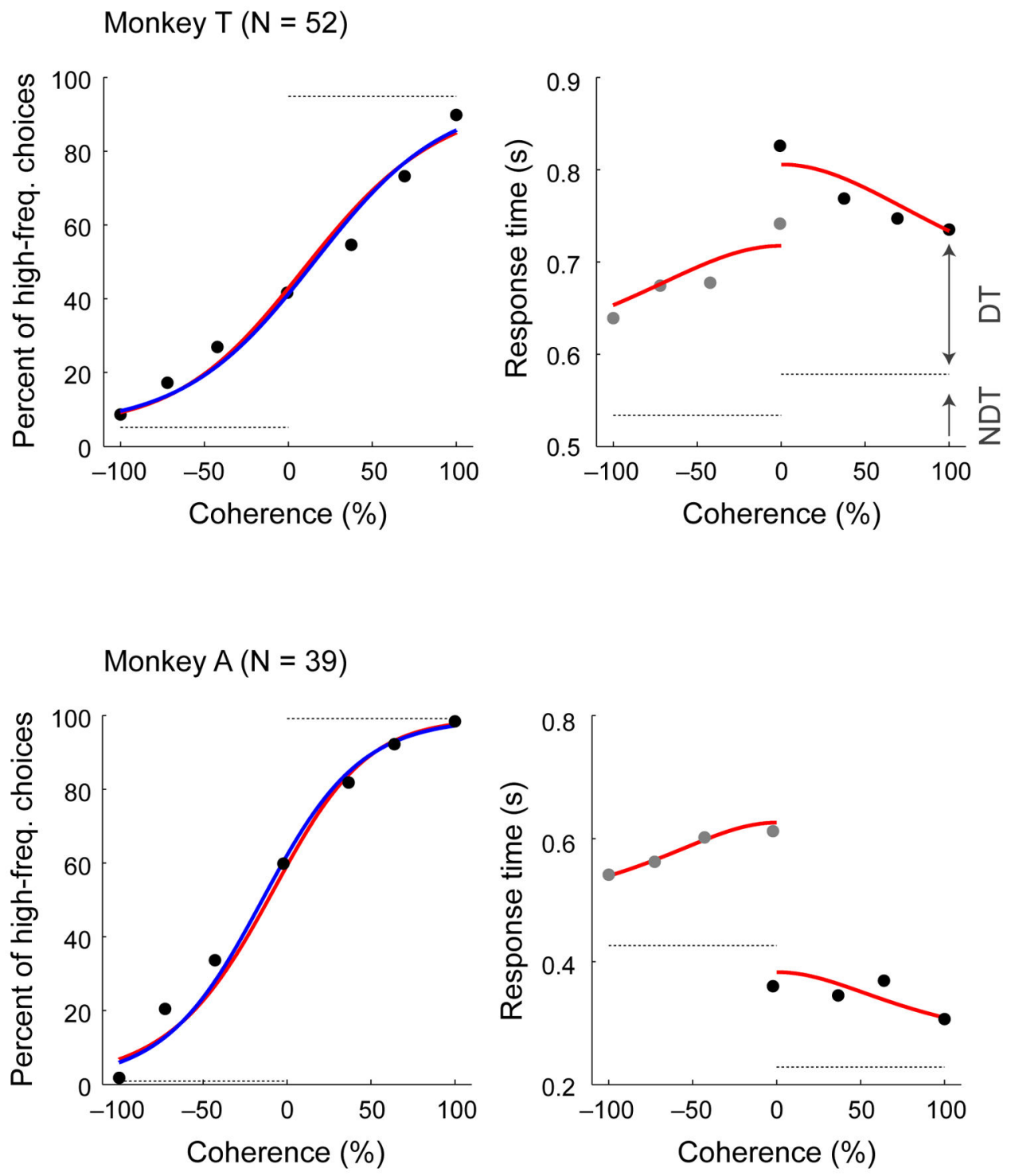

Figure 2. Psychophysical performance on the low-high task

Psychometric (left) and chronometric (right) functions for Monkey T (top) and Monkey A (bottom). Psychometric functions are plotted as the percentage of trials in which monkey chose "high frequency" as a function of signed coherence, where larger negative/positive coherence values indicate more low/high frequency tone bursts. The horizontal dashed grey lines on the psychometric plots indicate lapse rate (errors for strong stimuli, presumably reflecting lapses in attention or inappropriate application of the decision-motor mapping, which were estimated from logistic-model fits indicated as solid blue curves). Chronometric functions are plotted as the mean RT, which was the time interval between stimulus onset and onset of joystick movement, on correct trials as a function of signed coherence. Grey dots are low-frequency choices, and black dots are high-frequency choices. Solid red curves are simultaneous fits of both psychometric and chronometric data to a drift-diffusion model $(\mathrm{DDM})^{18 \_20},{ }^{29}{ }^{33}$. The horizontal dashed grey lines on the chronometric plots indicate choice-dependent non-decision times (NDT) estimated by the DDM fits. Decision times 
(DT) were estimated as the difference between the trial-specific RT and the choice-specific NDT. 
a

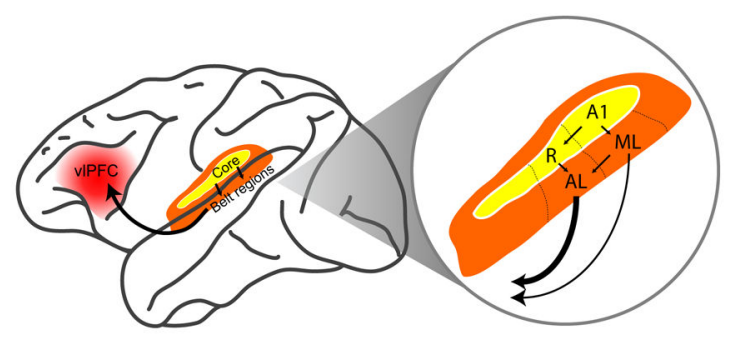

b
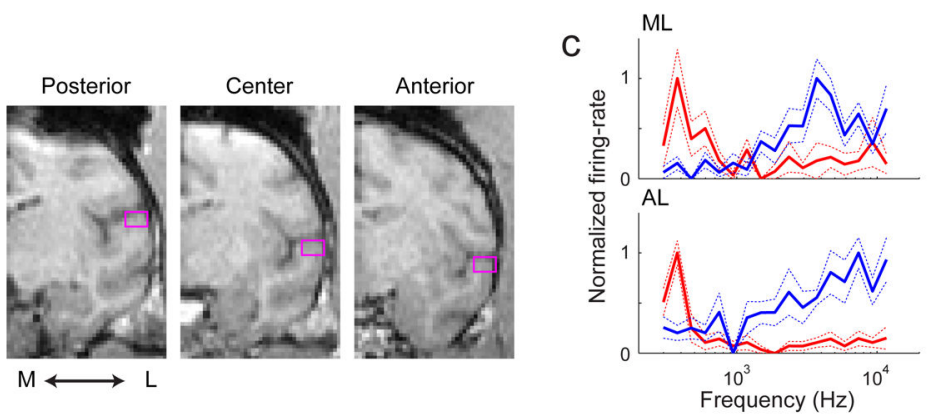

d
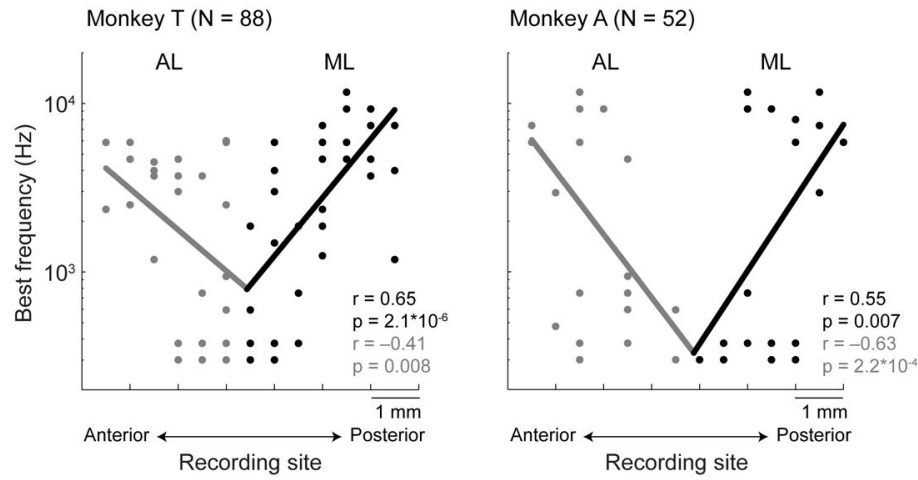

Figure 3. Recording locations and ML/AL tonotopy a, Schematic of the ventral auditory pathway. b, Coronal MRI sections of monkey A's brain at the level of the superior temporal gyrus. The purple squares indicate targeted locations of ML and AL. c, Examples of tuned frequency-response profiles from ML (top) and AL (bottom). Each colored line represents responses from a different neuron. Solid lines indicate mean values; dotted lines indicate s.e.m. d, Reconstructions of ML and AL tonotopy. The preferred frequency of ML neurons increased at more posteriorly located sites, whereas those of AL neurons increased at more anterior locations. The ML-AL border was identified from the location of the frequency-gradient reversal. The solid lines are linear regressions that relate recording location with preferred frequency in ML and AL. 
a
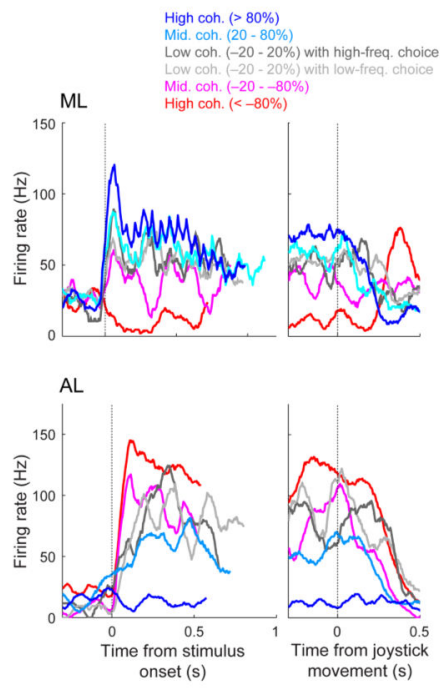

b
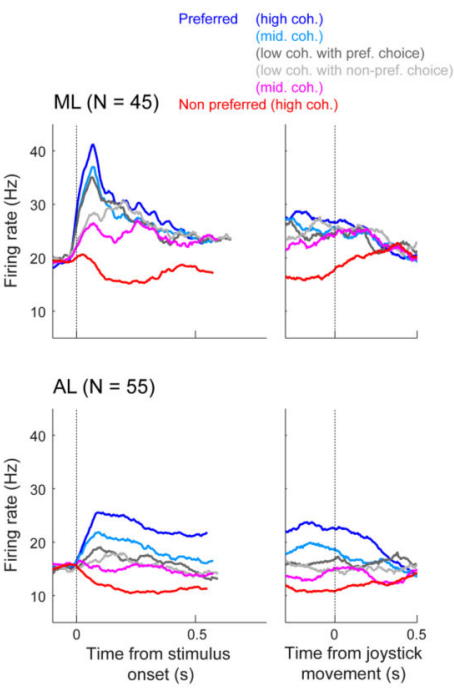

Figure 4. Neuronal sensitivity to stimulus frequency and coherence in ML (top) and AL (bottom) a, Single-neuron examples of responses from correct trials. Color corresponds to stimulus coherence. Grey lines show low-coherence responses separated by choice, as indicated. Left panels show activity aligned to stimulus onset. Right panels show activity aligned to the onset of joystick movement. b, Population histograms from correct trials, plotted as in a except that coherence is encoded with respect to each neuron's preferred and non-preferred frequency (see legend). 

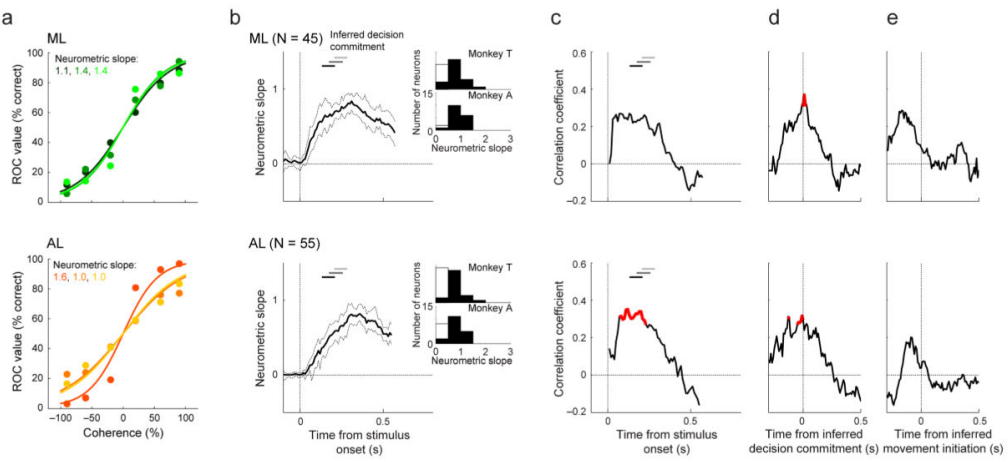

Figure 5. Relationship between neurometric and psychometric sensitivity for ML (top) and AL (bottom)

a, Example neurometric curves. Different curves and their corresponding neurometric slopes (an index of sensitivity) are shown in different colors. The curves were obtained from neural data elicited at the time of the peak of the correlation between neurometric and behavioral sensitivity (see panel c). b, Time courses of average neurometric slopes. The neurometric slope was calculated using 300-ms bins (with 10-ms increments) relative to stimulus onset. This relatively large bin size was needed to obtain reliable slope measurements and likely exaggerates the apparently gradual rise in sensitivity following stimulus onset. Thick/dashed lines show median/95\%-confidence intervals across individual neurons. Insets show the distributions of neurometric slopes calculated from individual neurons using firing rates measured between stimulus onset and the inferred time of the decision commitment for each trial (i.e., the end of the decision time plus an additional $50 \mathrm{~ms}$ to account for the sensory latency), per monkey; black bars indicate $H_{0}$ : slope $>0, p<0.05$ (one-tailed permutation test). c, $\mathbf{d}$, e, Time-dependent correlations between neuron-by-neuron neurometric slope and the simultaneously measured psychometric slope, plotted relative to stimulus onset (c), the inferred time of the decision commitment $(\mathbf{d})$, and the inferred time of movement initiation (e). Significant regression coefficients are colored red (Spearman correlation coefficient, $p<0.05)$. In $\mathbf{b}$ and $\mathbf{c}$, the horizontal bars represent the range of the inferred times of decision commitment, for high $(<-80 \%$ and $>+80 \%$, black), middle $(-80 \%$ to $-20 \%$ and $+80 \%$ to $+20 \%$, dark gray), and low ( $-20 \%$ to $+20 \%$, light gray) coherence. 

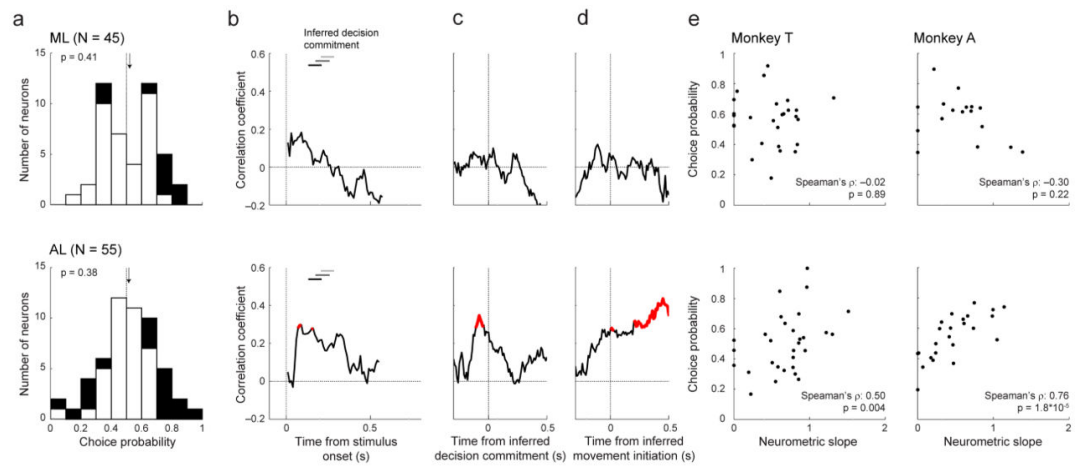

Figure 6. Relationship between choice probability and neurometric sensitivity for ML (top) and AL (bottom)

a, Distributions of choice probabilities calculated from firing rates between stimulus onset and the inferred time of the decision commitment (i.e., the end of the decision time plus an additional $50 \mathrm{~ms}$ ). Black bars indicate $H_{0}$ : choice probability $\nexists .5, p<0.05$ (two-tailed permutation test). Arrows indicate median values. b, c, d, Time-dependent correlations between neuron-by-neuron choice probability and neurometric sensitivity (i.e., slope), plotted relative to stimulus onset (b), the inferred time of the decision commitment (c), and the inferred time of movement initiation (d). Significant regression coefficients are highlighted in red (Spearman correlation coefficient, $p<0.05$ ). The horizontal bars represent the range of the inferred times of the decision commitment, for high $(<-80 \%$ and $>+80 \%$, black), middle ( $-80 \%$ to $-20 \%$ and $+80 \%$ to $+20 \%$, dark gray), and low $(-20 \%$ to $+20 \%$, light gray) coherence. e, Scatterplot examples showing the correlation between neurometric slope and choice probability for each monkey. These plots were generated from the time point with the largest correlation in AL for each monkey. 

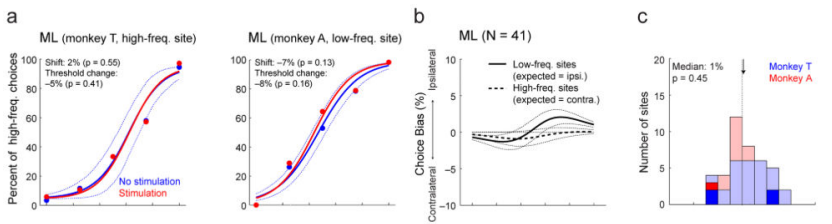

d
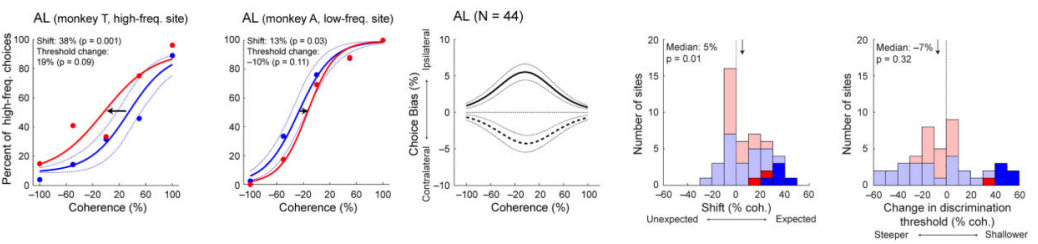

Figure 7. Effect of microstimulation on behavioral performance during the low-high task for ML (top) and AL (bottom)

a, Single-site examples of microstimulation effects on psychometric performance.

Psychometric functions are plotted as in Fig. 2. Red/blue symbols are for data from trials with/without microstimulation. Solid lines are logistic fits, computed separately for the two conditions. Dotted lines are $95 \%$ confidence intervals of the non-microstimulation trials that were calculated by a bootstrap procedure ${ }^{32}$. b. Average effects of microstimulation with respect to low-tone sites, which were expected to bias behavior towards more low-tone choices (corresponding to hand movements to the right, or ipsilateral to the targeted brain hemisphere; solid lines), and high-tone sites, which were expected to bias behavior towards more high-tone choices (corresponding to hand movements to the left, or contralateral to the targeted brain hemisphere; dashed lines). Solid lines indicate the median difference in choices for the given coherence for trials with versus without microstimulation. Dotted lines indicate bootstrapped $1^{\text {st }}$ and $3^{\text {rd }}$ quartiles of the median difference. $\mathbf{c}, \mathbf{d}$, Distributions of session-by-session effects of microstimulation on choice bias (c) and discrimination threshold (d) from logistic fits. The "expected" direction for choice bias is toward choices associated with the given site's preferred frequency, whereas "unexpected" is toward those associated with the non-preferred frequency. The data in blue is from monkey $\mathrm{T}$ and the data in red is from monkey A; dark bars indicate sites with significant $(p<0.05$; one-tailed permutation test) microstimulation effects. Arrows indicate median values; labels indicate result of two-tailed Wilcoxon rank test for $H_{0}$ : median $=0$. 